\title{
Caracterização de pacientes diabéticos tipo 2 em uso recente de insulina: estilo de vida e sintomas depressivos
}

\author{
Characterization of type 2 diabetic patients on recent insulin use: lifestyle and \\ depressive symptoms
}

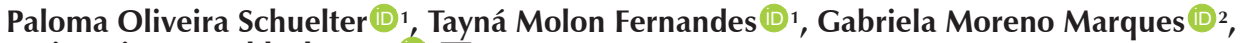 \\ Betine Pinto Moehlecke Iser $(\mathbb{D})^{2} \square$ \\ ' Faculdade de Medicina, Universidade do Sul de Santa Catarina. Tubarão, Santa Catarina, Brasil. \\ 2 Programa de Pós-Graduação em Ciências da Saúde, Universidade do Sul de Santa Catarina. Tubarão, Santa Catarina, Brasil.
}

Como citar este artigo (How to cite this article):

Schuelter PO, Fernandes TM, Marques GM, Moehlecke BP. Caracterização de pacientes diabéticos tipo 2 em uso recente de insulina: estilo de vida e sintomas depressivos (Characterization of type 2 diabetic patients on recent insulin use: lifestyle and depressive symptoms). Sci Med. 2019;29(3):e33463. https://doi.org/10.15448/1980-6108.2019.3.33463

\section{RESUMO}

OBJETIVO: Caracterizar os portadores de diabete melito tipo 2 que iniciaram recentemente o uso de insulina de acordo com estilo de vida e sintomas depressivos.

MÉTODO: Estudo transversal realizado com pacientes em uso recente de insulina, o qual foi determinado pelo cadastro realizado nas Estratégias de Saúde da Família de Tubarão-SC no período de janeiro de 2017 a agosto de 2018. Em visitas domiciliares, os pacientes responderam questões sobre estilo de vida, características da doença e o inventário de depressão de Beck (BDI).

RESULTADOS: A amostra foi composta por 38 pacientes com predomínio do sexo masculino (57,9\%), casados $(65,8 \%)$ e brancos $(89,5 \%)$, sendo a média de anos de estudo de 5,8 2,9 . Foram considerados ativos fisicamente no tempo livre $13,2 \%$ e $47,4 \%$ faziam restrição de carboidratos. Apresentavam sobrepeso $65,8 \%$ e obesidade $34,2 \%$. Os participantes receberam o diagnóstico de diabete melito tipo 2 , em média, há $8,9 \pm 7,5$ anos. Sobre os motivos que levaram ao uso de insulina, 92,1\% mencionaram o descontrole da doença. Quanto à classificação de sintomas depressivos, $47,3 \%$ apresentaram alguma alteração psicológica, considerando o BDI $>10$. A presença de sintomas depressivos foi maior nas mulheres $(\mathrm{p}=0,014)$.

CONCLUSÃO: Os pacientes com diabetes tipo 2 em uso recente de insulina apresentaram poucas mudanças no estilo de vida após o diagnóstico da doença. A maioria dos pacientes iniciou uso de insulina por descontrole da doença. A presença de sintomas depressivos foi maior nas mulheres e nos pacientes que relataram mudança da alimentação após o diagnóstico de diabete melito tipo 2.

DESCRITORES: Diabete melito; insulina; depressão.

\section{ABSTRACT}

AIMS: To characterize type 2 diabetes mellitus patients who have recently initiated treatment with insulin regarding lifestyle and depressive symptoms.

METHODS: Cross-sectional study carried out with patients in recent use of insulin, which was determined by the register held in the family health strategy, from January 2017 to August 2018. In home visits, the patients answered questions about lifestyle and characteristics of the disease, and Beck's Depression Inventory.

RESULTS: The sample consisted of 38 type 2 diabetic patients in recent use of insulin. There was a predominance of males (57.9\%), married $(65.8 \%)$ and white $(89.5 \%)$, the average years of study being $5.8 \pm 2.9 .13 .2 \%$ were considered physically active in leisure time, and only $47.4 \%$ were restricting carbohydrates. $65.8 \%$ were overweight and $34.2 \%$ were obese. The participants had type 2 diabetes mellitus diagnosis for $8.9 \pm 7.48$ years on average. Regarding the reasons that led to the use of insulin, $92.1 \%$ mentioned the lack of control of the disease. As for the classification of depressive symptoms, $47.3 \%$ had some psychological alteration. We verified that the presence of depressive symptoms was more prevalent in women $(\mathrm{p}=0,014)$.

CONCLUSION: Patients with type 2 diabetes on recent insulin use had few changes in lifestyle after being diagnosed with the disease. Most patients started using insulin due to lack of control of the disease. The presence of depressive symptoms was more prevalent in women and in patients who reported dietary changes after the diagnosis of type 2 diabetes mellitus.

KEYWORDS: Diabetes Mellitus; insulin; depression. 
Abreviaturas: DM, Diabetes Mellitus; BDI, Inventário de Depressão de Beck [do inglês, Beck Depression Inventory]; IMC, índice de massa corpórea.

\section{INTRODUÇÃO}

O diabete melito (DM) faz parte do grupo das doenças crônicas não transmissíveis [1], o qual tem importância significativa por sua crescente prevalência no Brasil e no mundo [2-3].A doença e suas complicações podem levar a prejuízos diretos para a economia e para vida social do portador da doença e sua família, além de prejuízos indiretos para todo o país, uma vez que resulta em redução dos anos de vida, incapacidades laborais, diminuição do poder de compra e ainda impacto financeiro sobre o sistema de saúde [1].

$\mathrm{O}$ aumento do número de portadores de DM se dá por múltiplos fatores, não apenas relacionados ao estilo de vida atual da população, como obesidade, sedentarismo, alimentação hedônica com excesso de carboidratos e gorduras, mas também por melhorias no tratamento das doenças crônicas, o que leva a um envelhecimento maior da população e maior sobrevida dos portadores da doença [4]. Segundo a Federação Internacional de Diabetes, o DM pode ser prevenido com práticas de vida saudável como exercícios físicos, perda de peso e alimentação saudável [3].

A estimativa nacional mais recente da Pesquisa Nacional de Saúde, realizada em 2013, verificou que $6,2 \%$ dos brasileiros adultos reportaram ter diagnóstico de diabetes [5]. Ainda, de acordo com a IDF, a cada ano, 7 milhões de pessoas desenvolvem diabetes e a cada 10 segundos morre uma pessoa vítima desta doença, sendo cerca de 2,9 milhões de óbitos causados pelo diabetes por ano no mundo [3].

Existem quatro subtipos de DM: DM tipo-1, DM tipo-2, DM gestacional e outros tipos de DM. O DM tipo-2 é o subtipo mais comum, agregando cerca de 90-95\% dos casos, e neste ocorre resistência progressiva à ação da insulina e por fim diminuição da sua produção pelas células beta pancreáticas [6].

O tratamento do DM tipo-2 deve ser individualizado de acordo com a clínica do paciente e com enfoque na qualidade de vida, mantendo os níveis de glicemia o mais próximo da normalidade e assim evitando as complicações da doença $[6,7]$. As complicações do DM podem ser: agudas - que são a hipoglicemia, a cetoacidose diabética e o estado hiperosmolar; e crônicas - que são divididas em microvasculares: retinopatia, neuropatia e nefropatia e macrovasculares: infarto agudo do miocárdio, acidente vascular encefálico e doença vascular periférica [8].

Medidas não farmacológicas, hipoglicemiantes orais e insulina são as possíveis formas de tratamento para o DM tipo-2 $[3,6,7]$. De acordo com a Sociedade Brasileira de Diabetes, a insulina deve ser introduzida no DM tipo-2 quando não se conseguiu atingir o controle glicêmico adequado com medidas não farmacológicas e hipoglicemiantes orais, ou em casos de aumento importante da glicemia, associados a perda de peso ou quando ocorrem intercorrências médicas [7]. As insulinas podem ser análogas e humanas, essas últimas, através do Sistema Único de Saúde, são disponibilizadas gratuitamente para os portadores de DM que necessitam de seu uso [9].

O DM tem sido associado com o aumento de sintomas depressivos e isso pode influenciar no controle metabólico e na adaptação do paciente à sua doença, podendo gerar o aparecimento de complicações decorrentes do DM. A depressão está ligada a reações e acontecimentos da vida de uma pessoa e pode ser agravada com a presença de uma doença crônica como o diabetes [10]. Estudos apontam que portadores de DM tipo-2 tem maior prevalência de depressão em relação a pessoas que não possuem a doença $[10,11]$.

Neste contexto, é importante conhecer as características dos pacientes que possuem a necessidade de iniciar o uso da insulina para o controle adequado da doença, considerando o aumento global da incidência de DM tipo-2 e o consequente aumento do número de usuários de insulina. Ainda, de acordo com informações repassadas pela secretaria de saúde do município de Tubarão-SC, tem ocorrido um aumento do número de pacientes com DM tipo-2 insulinodependentes, mesmo em estágios iniciais da doença, no entanto os motivos que levam de fato esses pacientes a necessitar de insulina são ainda desconhecidos. Assim, o objetivo desse estudo é caracterizar os portadores de DM tipo-2 que iniciaram recentemente o uso de insulina, de acordo como estilo de vida e a presença de sintomas depressivos.

\section{MÉTODOS}

Trata-se de um estudo observacional do tipo transversal. $\mathrm{O}$ estudo avaliou pacientes em uso recente de insulina em uma cidade do Sul de Santa Catarina. O uso recente foi determinado pelo cadastro dos pacientes com DM tipo-2 em uso de insulina realizado junto às equipes de Estratégia de Saúde da Família, no período de janeiro de 2017 a agosto de 2018. O cadastro é 
necessário para que os pacientes do Sistema Único de Saúde recebam a medicação.

Foram incluídos pacientes com DM tipo-2, adultos $(\geq 18$ anos) que iniciaram insulinização pelo Sistema Único de Saúde de janeiro de 2017 a agosto de 2018, que estavam em sua residência no dia da visita e que após convite e orientação quanto aos objetivos do estudo, aceitaram participar e preencheram o Termo de Consentimento Livre e Esclarecido. Foram excluídos os pacientes que apresentaram alguma condição física ou mental que impossibilitasse a entrevista ou o preenchimento do documento. O estudo foi aprovado pelo Comitê de Ética em Pesquisa da Unisul, sob parecer $\mathrm{n}$ - 2.516.371 e segue os preceitos da resolução 466/2012 do Conselho Nacional de Saúde.

A coleta de dados foi feita de março a agosto de 2018 por meio de visitas domiciliares junto às agentes comunitárias das estratégias de saúde da família do município, por um dos membros da equipe de pesquisa. A identificação dos pacientes em uso recente de insulina foi realizada com auxílio da enfermeira responsável por cada serviço e pelas agentes comunitárias de saúde de cada microárea.

Previamente à aplicação da pesquisa, na primeira fase da coleta de dados, realizou-se um estudo piloto, com pacientes com diabetes tipo 2 em uso de insulina cadastrados há mais tempo, anteriormente ao período do estudo, a fim de testar a aplicabilidade do questionário e a logística da coleta.

Os participantes do estudo, após preencher o termo de consentimento livre e esclarecido, responderam, inicialmente, perguntas sobre características sociodemográficas, questões sobre estilo de vida e caracterização clínica da doença.

As questões sobre estilo de vida foram baseadas no algoritmo da Sociedade Brasileira de Diabetes de 2017 e nas questões utilizadas no Sistema de vigilância de fatores de risco e proteção para doenças crônicas por inquérito telefônico realizado pelo Ministério da Saúde, de forma a constituir indicadores específicos $[7,12]$. Para a classificação da população estudada segundo nível de atividade física realizada, considerou-se ativo no lazer o indivíduo que pratica pelo menos 150 minutos de atividade física moderada por semana nos últimos três meses considerando duração e intensidade do exercício físico [12]. Já o consumo de álcool foi considerado abusivo, para pacientes diabéticos adultos, quando homens referiram a ingestão de mais de duas doses diárias e mulheres mais de uma dose diária (considera-se uma dose uma taça de vinho ou uma lata pequena de cerveja) [7]. Quanto ao estado nutricional, considerou-se sobrepeso quando o índice de massa corpórea (IMC) foi entre $25-29,9 \mathrm{~kg} / \mathrm{m}^{2}$ e obesidade $\mathrm{IMC} \geq 30 \mathrm{~kg} / \mathrm{m}^{2}[12]$.

Para avaliação da presença de sintomas depressivos, foi aplicado o Inventário de Depressão de Beck (BDI), traduzido e validado para a população adulta brasileira [13]. O BDI é um instrumento validado, estruturado, composto de 21 categorias de sintomas e atitudes, que descrevem manifestações comportamentais cognitivas afetivas e somáticas da depressão. Cada categoria contém quatro ou cinco alternativas que expressam níveis de gravidade dos sintomas depressivos. A pontuação para cada categoria varia de zero a três, sendo zero a ausência dos sintomas depressivos e três a presença dos sintomas mais intensos. $\mathrm{Na}$ dependência da pontuação total, os escores de até 9 pontos significam ausência de depressão ou sintomas depressivos mínimos; a partir de 10 pontos considera-se presença de alteração psicológica, sendo de 10 a 18 pontos, estado depressivo leve; de 19 a 29 pontos, estado depressivo moderado; e, de 30 a 63 pontos, estado depressivo grave. A classificação também pode ser baseada no ponto de corte de 21 pontos ou mais, a partir do qual se considera a existência de depressão clinicamente significativa [13].

A variável dependente do estudo foi o uso recente de insulina, e os motivos que levaram a esse uso, os quais foram avaliados de acordo com características epidemiológicas e clínicas dos pacientes (variáveis independentes).

Os dados coletados foram inseridos no programa Epi-Info 3.5.4, no qual foram organizadas e analisadas as informações coletadas, e a análise foi complementada no programa Stata 12.0. As variáveis quantitativas foram descritas por meio de medidas de tendência central e dispersão dos dados, segundo a normalidade dos dados. As variáveis qualitativas descritas por meio de frequência absoluta e percentual. As diferenças nas proporções foram testadas pelo teste de qui-quadrado ou exato de Fisher, e diferenças de médias pelo teste $t$ de Student, ou teste U de Mann-Whitney para comparação de medianas, conforme adequação dos dados. A medida de associação utilizada foi a razão de prevalência (RP), com intervalo de confiança (IC) de $95 \%$. O nível de significância estatística adotado foi de $5 \%$ (valor de $\mathrm{p}<0,05$ ).

\section{RESULTADOS}

Entre os 40 pacientes em uso recente de insulina identificados nas unidades de saúde do município de Tubarão, foram excluídos dois pacientes, um por apresentar déficit intelectual e um por ser cego, o 
que os impossibilitava de responder às perguntas $\mathrm{e}$ assinar o termo de consentimento livre e esclarecido, respectivamente. A amostra do estudo foi composta por 38 pacientes diabéticos tipo 2 em uso recente de insulina. A mediana de idade foi 65,5 anos (amplitude interquartil $=57-70$ ), variando de 28 a 79 anos; $63,2 \%$ da amostra tinha 60 anos ou mais. Predominou pacientes do sexo masculino $(57,9 \%)$, casados $(65,8 \%)$ e brancos $(89,5 \%)$, com até 8 anos de estudo $(92,1 \%)$, sendo a média de anos de estudo de 5,8 2,9 .

Na Tabela 1, são apresentados dados relativos ao estilo de vida dos pacientes estudados. Grande parte dos pacientes são considerados inativos e, para os que relataram realizar atividade física, a caminhada na rua foi a principal modalidade praticada $(73,3 \%$ dos praticantes). Entre aqueles que relataram mudança da alimentação após diagnóstico de DM, apenas $18(47,4 \%)$ pacientes faziam restrição de carboidratos.

De seis $(15,7 \%)$ pacientes que relataram consumo de álcool, predominou o uso 1 a 2 vezes por semana e desses, três pacientes faziam uso abusivo de álcool. Três $(7,8 \%)$ pacientes eram tabagistas, com uma média de 40 anos de fumo e consumo de 16,6 $\pm 5,7$ cigarros por dia. Outros $23(60,5 \%)$ pacientes são ex-fumantes, com uma média de 28,4 $\pm 14,5$ anos de fumo. Apenas um paciente interrompeu o uso após diagnóstico de DM (Tabela 1).

Tabela 1. Estilo de vida de pacientes com DM2 em uso recente de insulina cadastrados em unidade de saúde de um município do Sul de Santa Catarina.

\begin{tabular}{lcc}
\hline Variável & $\begin{array}{c}\text { Frequência }(\mathbf{n = 3 8}) \\
\mathbf{n}\end{array}$ & \% \\
\hline Realizou exercício últimos 3 meses & 15 & 39,5 \\
Tipo exercício ( $\mathrm{n}=15)$ & & \\
$\quad$ Caminhada na rua & 11 & 73,3 \\
$\quad$ Caminhada na esteira & 2 & 13,3 \\
$\quad$ Musculação & 2 & 13,3 \\
$\quad$ Ginástica em geral & 2 & 13,3 \\
$\quad$ Bicicleta & 3 & 20,0 \\
Nível de atividade física & & \\
Inativo & 24 & 63,2 \\
Insuficientemente ativo & 9 & 23,7 \\
Ativo & 5 & 13,2 \\
Mudou Alimentação após DM & 23 & 60,5 \\
Consumo de álcool & 6 & 15,8 \\
Periodicidade do consumo de álcool & & \\
(dias/semana) (n=6) & & \\
$\quad$ 1 a 2 dias & & 66,7 \\
todos os dias & 4 & 16,7 \\
$\quad$ menos de 1 dia por semana. & 1 & 16,7 \\
\hline
\end{tabular}

$\mathrm{DM}=$ Diabetes mellitus
Na Tabela 2, são apresentados os dados sobre estado nutricional e características clínicas da doença. A perda de peso após o diagnóstico de DM foi relatada por $20(52,6 \%)$ pacientes, sendo que a média de perda de peso foi $12,5 \pm 11,3 \mathrm{~kg}$. Mesmo assim, a média de IMC dos pacientes diabéticos tipo II, foi de $29,9 \pm 9,9 \mathrm{~kg} / \mathrm{m}^{2}$, dos quais $12(31,6 \%)$ tinham sobrepeso e $13(34,2 \%)$ obesidade.

Tabela 2. Características clínicas de pacientes com DM tipo-2 em uso recente de insulina cadastrados em unidades de saúde de um município do Sul de Santa Catarina.

\begin{tabular}{|c|c|c|}
\hline Variável & Frequência $(n=38)$ & $\%$ \\
\hline \multicolumn{3}{|l|}{ IMC $\left(\mathrm{kg} / \mathrm{m}^{2}\right)$} \\
\hline 19 a 24,99 & 13 & 34,2 \\
\hline 25 a 29,99 a & 12 & 31,6 \\
\hline 30 a $34,99^{\text {b }}$ & 6 & 15,8 \\
\hline 35 a 39,99 b & 3 & 7,9 \\
\hline$\geq 40^{\mathrm{b}}$ & 4 & 10,5 \\
\hline \multicolumn{3}{|l|}{$\begin{array}{l}\text { Quantidade de peso perdido após } \\
\text { diagnóstico }(n=20)\end{array}$} \\
\hline$\leq 5 \mathrm{~kg}$ & 7 & 35,0 \\
\hline 6 a $10 \mathrm{~kg}$ & 6 & 30,0 \\
\hline$>10 \mathrm{~kg}$ & 7 & 35,0 \\
\hline \multicolumn{3}{|l|}{ Tempo de DM } \\
\hline$\leq 5$ anos & 16 & 42,1 \\
\hline$>5$ anos & 22 & 57,9 \\
\hline Usa hipoglicemiantes & 31 & 81,5 \\
\hline \multicolumn{3}{|l|}{ Hipoglicemiantes em uso $(n=31)$} \\
\hline Metformina & 17 & 54,8 \\
\hline Metformina e glibenclamida & 9 & 29,0 \\
\hline Metformina e inibidor DPP4 & 2 & 6,5 \\
\hline Metformina e glimepirida & 2 & 6,5 \\
\hline Inibidor DPP4 & 1 & 3,2 \\
\hline \multicolumn{3}{|l|}{ Motivo do uso de insulina } \\
\hline DM não controlada & 35 & 92,1 \\
\hline Efeito colateral hipoglicemiante oral & 1 & 2,6 \\
\hline Hepatopatia & 1 & 2,6 \\
\hline Nefropatia & 1 & 2,6 \\
\hline \multicolumn{3}{|l|}{ Tempo uso insulina } \\
\hline$\leq 6$ meses & 22 & 57,9 \\
\hline$>6$ meses & 16 & 42,1 \\
\hline Outras doenças (além do DM) & 33 & 86,8 \\
\hline Hipertensão Arterial Sistêmica & 25 & 65,8 \\
\hline Dislipidemia & 12 & 31,6 \\
\hline Doença cardiaca & 8 & 21,0 \\
\hline Doenças da tireóide ${ }^{c}$ & 5 & 13,2 \\
\hline Depressão ou ansiedade & 5 & 13,2 \\
\hline Artrose ou artrite & 3 & 7,9 \\
\hline Oftalmopatia & 2 & 5,3 \\
\hline Outras $^{d}$ & 4 & 18,4 \\
\hline
\end{tabular}

a Sobrepeso: IMC entre $25-29,9 \mathrm{~kg} / \mathrm{m}^{2}$; ${ }^{\mathrm{b}}$ Obesidade: IMC $\geq 30 \mathrm{~kg} / \mathrm{m}^{2}$. ${ }^{\mathrm{c}}$ Doenças da tireóide: hipotireoidismo $(n=4)$ e hipertireoidismo $(n=1)$; d Outras doenças relatadas incluem osteoporose, labirintite, hepatopatia e nefropatia, relatadas por um paciente cada. DM: Diabetes mellitus; DPP4: Enzima dipeptidil peptidase-4; IMC: Índice de massa corporal. 
Os pacientes DM tipo-2 em uso de insulina receberam o diagnóstico da doença, em média, há $8,9 \pm 7,5$ anos. O uso de hipoglicemiantes orais, previamente ao uso de insulina, foi feito por $33(86,8 \%)$ pacientes, sendo que, nesses, foi predominante o uso prévio da associação de metformina e glibenclamida $(48,5 \%)$, seguido do uso isolado de metformina $(39,3 \%)$. O uso concomitante de hipoglicemiantes orais com insulina no momento da entrevista foi relatado por $31(81,5 \%)$ pacientes, sendo o uso isolado de metformina o mais frequente (Tabela 2).

Sobre os motivos que levaram ao uso de insulina, $35(92,1 \%)$ pacientes mencionaram o descontrole da doença. A média de tempo do uso de insulina, desde seu início, foi de $6,9 \pm 5,8$ meses. A maioria dos pacientes apresentou outra doença além do diabetes, sendo a mais prevalente a hipertensão arterial sistêmica em $25(65,8 \%)$ pacientes (Tabela 2$)$.

Tabela 3. Médias e níveis de pontuação de sintomas depressivos através do BDI de pacientes com DM2 insulinodependentes de uma cidade do Sul de Santa Catarina.

\begin{tabular}{|c|c|c|}
\hline Variável & média $\pm D P$ & Min-Máx \\
\hline Tristeza & $0,86 \pm 0,81$ & 0 e 3 \\
\hline Pessimismo & $0,73 \pm 0,85$ & 0 e 3 \\
\hline Fracasso passado & $0,31 \pm 0,61$ & 0 e 2 \\
\hline Perda de prazer & $0,78 \pm 0,74$ & 0 e 2 \\
\hline Sentimento de culpa & $0,26 \pm 0,51$ & 0 e 2 \\
\hline Sentimento de punição & $0,39 \pm 0,82$ & 0 e 3 \\
\hline Autoestima & $0,39 \pm 0,71$ & 0 e 3 \\
\hline Autocrítica & $0,28 \pm 0,56$ & 0 e 2 \\
\hline Pensamentos desejos suicidas & $0,26 \pm 0,68$ & 0 e 3 \\
\hline Choro & $0,63 \pm 0,97$ & 0 e 3 \\
\hline Irritabilidade & $0,65 \pm 0,81$ & 0 e 2 \\
\hline Interação social & $0,63 \pm 0,78$ & 0 e 2 \\
\hline Indecisão & $0,42 \pm 0,59$ & 0 e 2 \\
\hline Mudança imagem corporal & $0,34 \pm 0,50$ & 0 e 3 \\
\hline Disposição & $1,07 \pm 1,14$ & 0 e 3 \\
\hline Alteração sono & $0,76 \pm 1,07$ & 0 e 3 \\
\hline Cansaço ou fadiga & $0,76 \pm 0,81$ & 0 e 3 \\
\hline Alteração apetite & $0,15 \pm 0,43$ & 0 e 2 \\
\hline Perda de peso & $0,42 \pm 0,91$ & 0 e 3 \\
\hline Preocupação somática & $0,71 \pm 0,83$ & 0 e 3 \\
\hline Perda de interesse por sexo & $0,73 \pm 1,00$ & 0 e 3 \\
\hline \multicolumn{3}{|l|}{ Pontuação BDI } \\
\hline$<10$ (sem depressão) & 20 & 52,6 \\
\hline 10 a 18 (ED leve) & 11 & 28,9 \\
\hline 19 a 29 (ED moderado) & 4 & 10,5 \\
\hline$\geq 30$ (ED grave) & 3 & 7,9 \\
\hline
\end{tabular}

$\mathrm{BDI}=$ Inventário de Depressão de Beck; $\mathrm{DP}=$ Desvio-padrão; $\mathrm{ED}=$ Estado depressivo.
Quanto à classificação de sintomas depressivos por meio do BDI, $18(47,4 \%)$ apresentaram pontuação compatível com alguma alteração psicológica, sendo que a média do BDI foi $11,1 \pm 10,6$ pontos. A existência de depressão clinicamente significativa foi verificada em cinco $(13,2 \%)$ pacientes (Tabela 3 ).

Comparando o tempo de diagnóstico com o tempo de uso de insulina, os pacientes apresentaram um tempo mediano com a doença de 8,5 anos antes de precisar do uso de insulina, embora com bastante variação (de 0 a 28,8 anos).

A Tabela 4 expõe a análise do BDI com as características dos pacientes e da doença, em que se verificou que as mulheres apresentaram maior pontuação na escala de sintomas depressivos. As mulheres também apresentaram aproximadamente duas vezes maior frequência de qualquer alteração

Tabela 4. Relação de score do BDI com características sociodemográficas e clínicas dos pacientes com DM2 em uso recente de insulina cadastrados em unidade de saúde de um município do Sul de Santa Catarina.

\begin{tabular}{|c|c|c|c|}
\hline Variável & Escore médio & DP & Valor de $p$ \\
\hline \multicolumn{4}{|l|}{ Sexo } \\
\hline masculino & 16 & 11,5 & 0,014 \\
\hline feminino & 7,5 & 8,5 & \\
\hline \multicolumn{4}{|l|}{ Escolaridade } \\
\hline$\leq 8$ anos & 11,17 & 10,91 & 0,93 \\
\hline$>8$ anos & 10,66 & 8,38 & \\
\hline \multicolumn{4}{|l|}{ Idade } \\
\hline$<60$ & 9,85 & 9,37 & 0,57 \\
\hline$\geq 60$ & 11,87 & 11,44 & \\
\hline \multicolumn{4}{|l|}{ Perdeu peso } \\
\hline não & 8,55 & 8,88 & 0,15 \\
\hline $\operatorname{sim}$ & 13,45 & 11,74 & \\
\hline \multicolumn{4}{|l|}{ Ativo no lazer } \\
\hline não & 11,18 & 10,74 & 0,94 \\
\hline $\operatorname{sim}$ & 10,8 & 11,09 & \\
\hline \multicolumn{4}{|c|}{ Mudou alimentação } \\
\hline não & 8,06 & 10,15 & 0,15 \\
\hline $\operatorname{sim}$ & 13,13 & 10,68 & \\
\hline \multicolumn{4}{|c|}{ Descontrole da glicemia } \\
\hline não & 1,5 & 2,12 & 0,19 \\
\hline $\operatorname{sim}$ & 11,66 & 1,5 & \\
\hline \multicolumn{4}{|c|}{ Tempo de uso de insulina } \\
\hline$\leq 6$ meses & 11,63 & 8,98 & 0,73 \\
\hline \multirow[t]{2}{*}{$>6$ meses } & 10,43 & 11,88 & \\
\hline & mediana & P25-P75 & valor de $p$ \\
\hline \multicolumn{4}{|c|}{ Tempo de diagnóstico } \\
\hline$\leq 5$ anos & 7,5 & $3-14$ & 0,43 \\
\hline$>5$ anos & 10 & $1-20$ & \\
\hline
\end{tabular}

BDI: Inventário de Depressão de Beck; DP: Desvio-padrão. P25-P75 = Amplitude interquartil (percentis 25 e 75) 
psicológica ( $\mathrm{RP}=2,75 ; \mathrm{IC} 95 \%: 1,31-5,76 ; \mathrm{p}=0,003)$ em comparação aos homens. Não foram verificadas diferenças na pontuação do BDI em relação a escolaridade e a idade, assim como com nível de atividade física, mudanças alimentares, perda de peso, tempo de diagnóstico e tempo de uso de insulina (Tabela 4). Considerando a presença de sintomas depressivos, para aqueles que apresentaram um escore $\geq 10$, verificou-se que os participantes que referiram ter realizado mudanças na alimentação após o diagnóstico de DM tiveram cerca de 1,7 vezes mais sintomas depressivos (RP =1,72; IC95\%: 1,00-1,98; $\mathrm{p}=0,039)$ em comparação àqueles que não relataram tais mudanças.

Os participantes que referiram o descontrole da doença como motivo do início do uso de insulina apresentaram, expressivamente, ainda que sem significância estatística, maiores médias do BDI $(11,7 \pm 10,7$ pontos $)$ em relação aos que referiram outros motivos (média 1,5 $\pm 2,1$ pontos) (Tabela 4 ).

\section{DISCUSSÃO}

Este estudo teve como objetivo principal caracterizar os portadores de DM tipo-2 em uso recente de insulina quanto ao estilo de vida e a presença de sintomas depressivos. Foi verificada associação positiva entre sexo feminino e sintomas depressivos, corroborando com resultados de Freitas, obtidos através de um estudo transversal, o qual mostrou que mulheres diabéticas possuem maior prevalência de depressão grave em comparação aos homens [14]. Ainda, uma coorte realizada na Holanda com pacientes diabéticos da atenção básica de saúde evidenciou maior incidência de qualquer grau de depressão nas mulheres, semelhante ao verificado em estudo que avaliou depressão não diagnosticado em pacientes DM tipo-2 $[11,15]$. Tais resultados podem ser explicados pela própria epidemiologia da doença, na qual mulheres apresentam duas vezes mais depressão que os homens [16].

Ainda no que diz respeito aos sintomas depressivos, apresentaram maior prevalência os pacientes que relataram mudança da alimentação após o diagnóstico de DM. Estudos indicam que os sintomas depressivos podem influenciar negativamente na adesão ao tratamento da doença e no controle glicêmico, o que pode ser explicado por sentimentos de ansiedade, medo e dificuldade de aceitação da doença em pacientes que possuem uma doença crônica, principalmente nas fases iniciais onde o paciente terá que se adaptar a mudanças na alimentação e demais práticas de estilo de vida, além de questões fisiológicas e de interação medicamentosa $[10,11,17]$. Da mesma forma, esse dado sugere que a necessidade de restrição alimentar devido à doença pode desencadear sentimentos de negação e frustração nessa população.

No que diz respeito ao motivo mencionado para o início da insulinização, a maioria dos pacientes relatou o descontrole da glicemia. Como os pacientes do presente estudo são provenientes do Sistema Único de Saúde, uma possível explicação para o alto índice de descontrole referido é o número limitado de opções de hipoglicemiantes orais disponibilizados, além da má adesão ao tratamento não medicamentoso, uma vez que mudanças do estilo de vida foram relatadas pela minoria dos pacientes. O comportamento sedentário, por exemplo, está presente na maioria dos diabéticos e pode ser ainda mais notável em pacientes insulinodependentes, a exemplo do verificado neste estudo, em que apenas cinco pacientes relataram ser efetivamente ativos no tempo livre, e dos dados de Leal et al., onde $83,3 \%$ não praticavam nenhuma atividade física [18-20]. A prática de 150 minutos semanais de atividade física é recomendada pela Organização Mundial de Saúde a toda população e, para pacientes diabéticos, segundo uma metanálise de ensaios clínicos randomizados, é o mínimo necessário para uma melhora do controle glicêmico [21-22].

Além disso, grande parte dos participantes do presente estudo tinha sobrepeso ou obesidade no momento da entrevista, o que, associado ao sedentarismo, dificulta o controle do DM por gerar maior resistência a ação da insulina e, consequentemente, faz com que o paciente necessite de uma maior dose da medicação $[6,23]$. Achados como esses expõem uma falha da atenção básica de saúde na aplicação de medidas de promoção e prevenção em saúde para conscientizar a população diabética sobre a importância de adotar um estilo de vida saudável, fundamental tanto para o controle da doença como para evitar suas complicações $[3,6,7]$. Vale ressaltar que, para isso, foi idealizado pelo Ministério da Saúde o "HIPERDIA" (sistema de cadastramento e acompanhamento de hipertensos e diabéticos), criado com intuito de acolher e orientar esses pacientes, contudo pouco aplicado na prática nas unidades de saúde onde a coleta de dados foi realizada [24].

No âmbito das doenças concomitantes ao DM, a maioria dos pacientes referiu hipertensão arterial sistêmica, corroborando com a literatura que mostra a notável associação das duas doenças, sendo um fator de agravo para esses pacientes [8,23]. Foram relatadas também a ocorrência de doença cardíaca, 
retinopatia e nefropatia, condições que podem estar relacionadas a complicações crônicas da doença, vista que os participantes do presente estudo tiveram aproximadamente nove anos de diagnóstico e pouca adesão a medidas não farmacológicas para controle da doença $[3,6,8]$.

Em relação ao perfil sociodemográfico, houve predomínio da população idosa (a partir de 60 anos), dado consistente com a epidemiologia do DM tipo-2 em que a prevalência aumenta consideravelmente com a idade $[2,3,25]$. Esta constatação levanta a um debate importante em relação a políticas de prevenção em saúde, visto que no Brasil e no mundo ocorre um aumento da expectativa de vida e por consequência aumento de doenças crônicas como o DM [1,3].

Observou-se que entre os diabéticos tipo 2 usuários de insulina, a maioria eram homens, sendo que tal dado contrapõe a literatura no que se refere a diagnóstico autorreferido de DM tipo-2, onde a maior prevalência é no sexo feminino, o que em geral está relacionado a maior frequência de mulheres nos serviços de saúde $[2,3,5]$. Assim, o maior uso de insulina em homens poderia indicar também que são eles os que têm menor adesão a medidas de controle da doença, necessitando do uso de insulina precocemente. Ainda, no estudo longitudinal de saúde do adulto brasileiro, aplicado em três capitais brasileiras e utilizando medidas bioquímicas, foi encontrada maior prevalência de diabetes em homens [26].

O presente estudo apresentou algumas limitações em relação à captação dos pacientes elegíveis ao estudo. Previamente a sua realização, o Núcleo de Vigilância Epidemiológica forneceu aos pesquisadores um cadastro dos novos pacientes em uso de insulina (a partir de janeiro de 2017). No entanto, ao iniciar as visitas domiciliares, notou-se uma discrepância no número de pacientes em uso de insulina recente pelo cadastro fornecido com os que realmente iniciaram uso no período citado. Isso ocorreu porque muitos pacientes foram cadastrados algum tempo depois de iniciar o uso, ou apresentavam mais de um cadastro.
Dessa forma, optou-se por utilizar o cadastro das agentes de saúde de cada unidade de saúde; porém pelo fato de haver microáreas ainda descobertas, não se obteve uma amostra real do número total de pacientes em uso recente de insulina.

Verificou-se nesse estudo que os pacientes com DM tipo-2 em uso recente de insulina apresentaram, em sua maioria, poucas mudanças para alcançarem um estilo de vida saudável. Os participantes referiram pouca prática de atividade física e pouco cuidado com alimentação, além de apresentarem alta prevalência de sobrepeso e obesidade. A presença de sintomas depressivos foi significativamente maior em mulheres, bem como para aqueles que relataram mudanças alimentares após o diagnóstico do DM. A maioria dos pacientes iniciou uso de insulina por descontrole da doença. Desta forma, nota-se a necessidade de aplicação de políticas públicas tanto de educação, quanto de apoio aos pacientes que possuem diabetes e outras doenças crônicas.

\section{NOTAS}

Apoio financeiro

Este estudo não recebeu apoio financeiro de fontes externas.

Declaração de conflito de interesses

Os autores declaram não haver conflitos de interesses relevantes ao conteúdo deste estudo.

\section{Contribuições dos autores}

Todos os autores fizeram contribuições substanciais para concepção, ou delineamento, ou aquisição, ou análise ou interpretação de dados; e redação do trabalho ou revisão crítica; e aprovação final da versão para publicação.

Disponibilidade dos dados e responsabilidade pelos resultados

Todos os autores declaram ter tido total acesso aos dados obtidos e assumem completa responsabilidade pela integridade destes resultados.

Agradecimentos

O presente trabalho foi realizado com apoio da Coordenação de Aperfeiçoamento de Pessoal Nível Superior - Brasil (CAPES).

\section{REFERÊNCIAS}

1. Alves CG, de Morais Neto OL. Trends in premature mortality due to chronic non communicable diseases in Brazilin Federal units. Ciênc Saúde Coletiva. 2015;20(3):641-54. https://doi.org/10.1590/1413-81232015203.15342014

2. Iser BPM, Vigo A, Duncan BB, Schmidt MI. Trends in the prevalence of self-reported diabetes in Brazilian capital cities and the Federal District, 2006-2014. Diabetol Metab Syndr. 2016;8:70. https://doi.org/10.1186/s13098-016$0185-\mathrm{x}$ 
3. International Diabetes Federation. IDF diabetes atlas [Internet]. 8th ed. Brussels: International Diabetes Federation; 2017 [capturado em 04 out. 2017]. Disponível em: https:/www.idf.org/diabetesatlas.

4. Malta DC, Iser BPM, Chueiri PS, Stopa SR, Szwarcwald CL, Schmidt MI, Duncan BB. Cuidados em saúde entre portadores de diabetes mellitus autorreferido no Brasil, Pesquisa Nacional de Saúde, 2013. Rev Bras Epidemiol. 2015;18(supl. 2):17-32. https://doi.org/10.1590/1980-5497201500060003

5. Iser BPM, Stopa SR, Chueiri PS, Szwarcwald CL, Malta DC, Monteiro HOC, Duncan BB, Schmidt MI. Prevalência de diabetes autorreferido no Brasil: resultados da Pesquisa Nacional de Saúde 2013. Epidemiol Serv Saúde. 2015;24(2):305-14. https://doi.org/10.5123/S1679-49742015000200013

6. American Diabetes Association. Standards of medical care in diabetes: 2019 [Internet]. Arlington: ADA; 2019 [capturado em 04 set. 2019]. Disponível em: https://professional.diabetes.org/content-page/standards-medical-carediabetes.

7. Sociedade Brasileira de Diabetes. Conduta terapeutica do diabetes tipo 2: algoritmo da SBD 2017. São Paulo: SBD; 2017.

8. Cortez DN, Reis IA, Souza DAS, Macedo MML, Torres HC. Complicações e o tempo de diagnóstico do diabetes mellitus na atenção primária. Acta Paul Enferm. 2015;28(3):250-5. https://doi.org/10.1590/1982-0194 201500042

9. Sociedade Brasileira de Diabetes. Revisão sobre análogos de insulina: indicações e recomendações para a disponibilização pelos serviços públicos de saúde [Internet]. São Paulo: SBD; 2011 [capturado em 20 out. 2017]. Disponível em: https:/www.diabetes.org.br/publico/images/pdf/posicionamento-sbd.pdf.

10. Ramos LBS, Santana CN, Araújo LLC, Jesus GP, Gois CF, Santos FL, Brito GM, Santos EF. Qualidade de vida, depressão e adesão ao tratamento de pessoas com diabetes. Rev Bras Ciênc Saúde. 2017;21(3):261-8. https://doi.org/ $10.22478 /$ ufpb.2317-6032.2017v21n3.29085

11. Alajmani DSA, Alkaabi AM, Alhosani MW, Folad AA, Abdouli FA, Carrick FR, Abdulrahman M. Prevalence of undiagnosed depression in patients with Type 2 Diabetes. Front Endocrinol. 2019;10:259. https://doi.org/10.3389/ fendo.2019.00259

12. Brasil. Ministério da Saúde. Vigitel Brasil 2016: vigilância de fatores de risco e proteção para doenças crônicas por inquérito telefônico. Brasília: Ministério da Saúde; 2017.

13. Gorenstein C, Andrade LHSG, Zuardi AW, editores. Escalas de avaliação clínica em psiquiatria e psicofarmacologia. São Paulo: Lemos; 2000.

14. Freitas CM. Diabetes mellitos tipo 2 e depressão: uma perspectiva psiconeuroimunológica [dissertação]. Juiz de Fora: Universidade Federal de Juiz de Fora; 2018.

15. Nefs G, Pouwer F, Denollet J, Pop V. The course of depressive symtoms in primary care patientes with type 2 diabetes: results from the Diabetes, Depression, Type D Personality Zuidoost-Brabant (DiaDDZoB) Study. Diabetologia. 2012;55(3):608-16. https://doi.org/10.1007/s00125-011-2411-2

16. Van de Velde S, Bracke P, Levecque K. Gender differences in depression in 23 European countries: crossnational variation in the gender gapin depression. SocSci Med. 2010;71(2):305-13. https://doi.org/10.1016/j. socscimed.2010.03.035

17. Zanoveli JM, Morais H, Dias IC, Schreiber AK, Souza CP de, Cunha JM. Depression associated with diabetes: from pathophysiology to treatment. Curr Diabetes Rev. 2016;12(3):165-78. https://doi.org/10.2174/1573399811666150 515125349

18. Lemes IR, Sui X, Turi-Lynch BC, Lee DC, Blair SN, Fernandes RA, Codogno JS, Monteiro HL. Sedentary behaviour is associated with diabetes mellitus in adults: findings of a cross-sectional analysis from the Brazilian National Health System. J Puplic Health (Oxf). 2018 Sept 27. https://doi.org/10.1093/pubmed/fdy169

19. Borba AKOT, Marques APO, Ramos VP, Leal MCC, Arruda IKG, Ramos RSPS. Fatores associados à adesão terapêutica em idosos diabéticos assistidos na atenção primária de saúde. Ciênc Saúde Coletiva. 2018;23(3):956-61. https://doi.org/10.1590/1413-81232018233.03722016

20. Leal PAMC, Bonfim CFA, Magalhães EIS, Silva RCS, Silva TM, Santos DP, Kochergin CN. Avaliação do controle glicêmico de pacientes diabéticos insulino-dependentes atendidos em unidade de saúde da família de Vitória da Conquista, Bahia. C\&D Rev Eletrônica Fainor. 2014;7(2):232-43. 
21. World Health Organization. More active people for a healthier world: the global action plan on physical activity 2018-2030 [Internet]. Geneva: WHO; 2019 [capturado em 20 out. 2018]. Disponível em: http://www.who.int/ncds/ prevention/physical-activity/gappa/.

22. Umpierre D, Ribeiro PA, Kramer CK, Leitão CB, Zucatti AT, Azevedo MJ, Gross JL, Ribeiro JP, Schaan BD. Physical activity advice only or structured exercise training and association with HbA1c levels in type 2 diabetes: a systematic review and meta-analysis. JAMA. 2011;305(17):1790-9. https://doi.org/10.1001/jama.2011.576

23. Maia RHS, Navarro AC. O exercício físico leve a moderado como tratamento da obesidade, hipertensão e diabetes. RBONE. 2017;66(11):393-402.

24. Brasil. Ministério da Saúde. Sistema de cadastramento e acompanhamento de Hipertensos e Diabéticos (HIPERDIA). Brasília: DATASUS; 2018.

25. Gusmai LF, Novato TS, Nogueira LS. The influence of quality of life in treatment adherence of diabetic patients: a systematic review. Rev Esc Enferm USP. 2015;49(5):839-46. https://doi.org/10.1590/S0080-623420150000500019

26. Schmidt MI, Hoffmann JF, Diniz MFS, Lotufo PA, Griep RH, Bensenor IM, Mill JG, Barreto SM, Aquino EML, Duncan BB. High prevalence of diabetes and intermediate hyperglycemia: The Brazilian Longitudinal Study of Adult Health (ELSA-Brasil). Diabetol Meta Syndr. 2014;6(1):123. https://doi.org/10.1186/1758-5996-6-123 